\title{
A New Approach to Estimate Fractal Dimension of Texture Images
}

\author{
André R. Backes and Odemir M. Bruno \\ Instituto de Ciências Matemáticas e de Computação (ICMC) \\ Universidade de São Paulo (USP) \\ Avenida do Trabalhador São-carlense, 400 \\ 13560-970 São Carlos SP Brazil \\ backes@icmc.usp.br, \\ bruno@icmc.usp.br
}

\begin{abstract}
One of the most important visual attributes for image analysis and pattern recognition is the texture. Its analysis allows to describe and identify different regions in the image through pixel organization, performing a better image description and classification. This paper presents a novel approach for texture analysis, based on calculation of the fractal dimension of binary images generated from a texture, using different threshold values. The proposed approach performs a complexity analysis as the threshold values changes, producing a texture signature which is able to characterize efficiently different texture classes. The paper illustrates the novel method performance on an experiment using Brodatz images.
\end{abstract}

Keywords: Fractal Dimension, Texture Analysis, Complexity.

\section{Introduction}

Texture is one of the most important visual attribute used for pattern recognition. It is characterized by repetition, exactly or with small variations, of a gray-scale or colors pattern over a region, and this pattern is straight connected with physical properties of the surface of the object 1123.

Its analysis allows to remark regions with same reflectance characteristics, and thus, the same colors in a determined combination of bands. This makes texture an excellent region descriptor, contributing for a more accurate process of image recognition, description and classification 1134.

Although its great importance in several applications, its analysis process demands a high level of sophistication and computer complexity. Moreover, it is a term which depends on our perception and still does not have a precise definition 14 .

Fractal dimension 567] is a measure of how complex an object is. In texture, this complexity is characterized by pixels organization, i.e., the complexity is straight connected to the visual aspect of the texture. So, fractal dimension allows to quantify a texture in terms of homogeneity, and makes possible a comparison between different textures [7/8]. 
In this work we propose a novel approach for texture characterization based on complexity analysis. Opposite to compute the complexity straight from the gray-scale texture, our main interest is to obtain a texture signature based on the complexity of binary images computed from the original texture using different thresholds. The complexity analysis is performed through application of the BoxCounting fractal dimension 9 906/7 over each binary image. The analysis of the obtained signature is performed using Linear Discriminant Analysis (LDA), which furnishes a reliable classification [1112].

The paper is divided as follows. A brief review of complexity and fractal dimension is presented in Section 2. In Section 3, we present a novel approach to apply the Box-Counting fractal dimension over gray-scale images in order to compute a texture signature. In Section 4 we set up an experiment to compare the performance of the proposed texture signature to 400 images of 40 different Brodatzs texture classes. Section [5] shows the results yielded by the proposed method, while in Section 6, the conclusions about the method are discussed.

\section{Fractal Dimension}

Complexity can be understood as a measure of how irregular an object is. Its is a term largely used on the literature, but, in spite of its importance, it lacks of an exact definition. An interesting way to estimate the complexity of an object is through fractal dimension.

Fractal dimension $(F D)$ is a measure of how irregular or complex an object is. Different from topological dimension, an integer value which describes the number of dimensions of the space where the object is inserted, fractal dimension is a fractionary value which describes how much of the space an object occupies [67.

One of the most known and used methods to estimate the fractal dimension of an object is the Box-Counting method. Its due to characteristics like easy implementation and simplicity of the computation involved in the method. Its computation is performed overlaying an image $A \in R^{2}$ with a grid of squares of size $r$ and counting the number of squares, $N(r)$, necessary to cover the object in the image (Figure 1) 9677.

The estimative of Box-Counting fractal dimension is based on relation between the size $r$ used in the grid of squares and the number of squares, $N(r)$, which contain a portion of the image object $A$ :

$$
F D=-\lim _{r \rightarrow 0} \frac{\log (N(r))}{\log (r)}
$$

From log-log plot of $r$ versus $N(r)$, we achieve a curve with $\alpha$ slope where

$$
F D=-\alpha
$$

is the Box-Counting fractal dimension of the object $A$ (Figure 2) 13710]. 

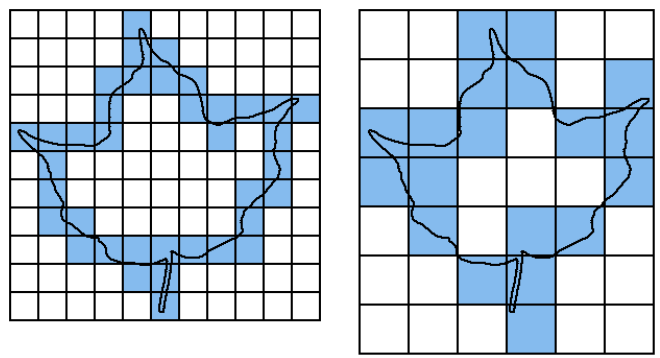

Fig. 1. Division of an image using the Box-Counting method for different $r$ values

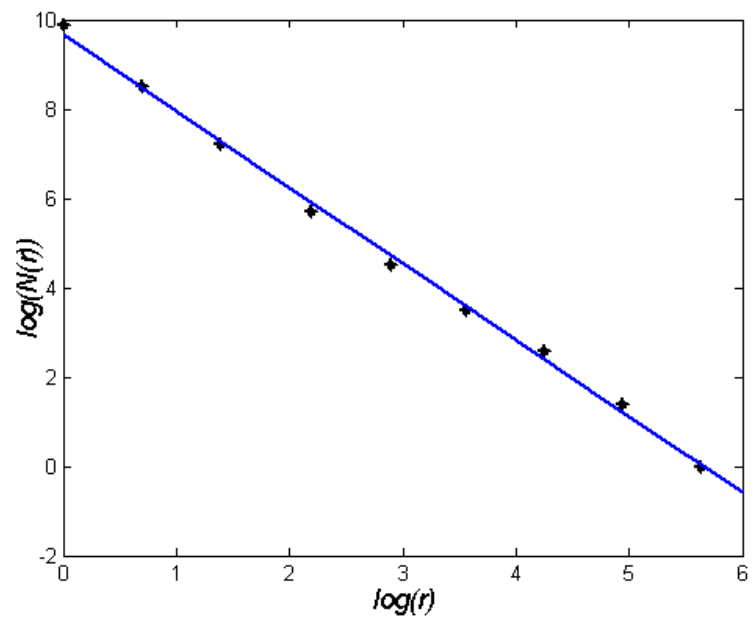

Fig. 2. Log-log plot of $r$ versus $N(r)$

\section{Proposed Method}

In this work we propose a novel approach for texture characterization based on complexity analysis. The basic idea of the method is to compute the fractal dimension of binary images. These images are generated as we apply different thresholds in the texture under analysis. So, its possible to compose a texture signature that describes how image complexity changes as we increase the threshold used.

Given a texture image $A \in R^{2}$ and a threshold value $T_{i}, T_{i} \in T$, a binary image $A_{T_{i}}$ is generated applying an $\delta_{T_{i}}$ transformation as follow:

$$
A_{T_{i}}=\delta_{T_{i}}(A)=\forall a \in A\left\{\begin{array}{l}
a_{T_{i}}=0, \text { if } a<T_{i} \\
a_{T_{i}}=1, \text { if } a \geq T_{i}
\end{array}\right.
$$

The complexity analysis is performed by applying the Box-Counting fractal dimension over the image $A_{T}$ : 


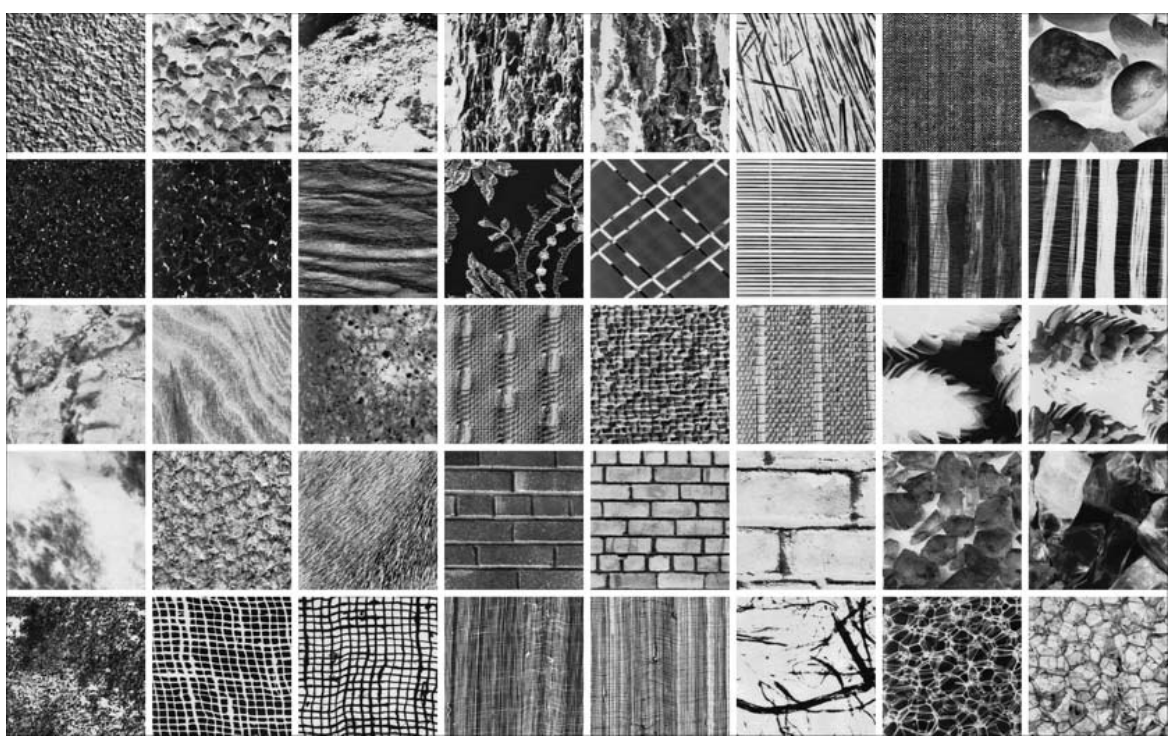

Fig. 3. One example of each of the 40 Brodatzs classes considered. Each image has 200 $\times 200$ pixels and 256 gray levels.

$$
F D_{T_{i}}=-\lim _{r \rightarrow 0} \frac{\log \left(N_{T_{i}}(r)\right)}{\log (r)}
$$

Considering different threshold values $T_{i}$, its is possible to compute a texture signature $\psi(A)$ :

$$
\psi(A)=\left[F D_{T_{1}}, F D_{T_{2}}, \ldots, F D_{T_{N}}\right] i \in 1 \ldots N,
$$

where $N$ is the number of thresholds considered for the texture characterization.

\section{Experiments}

In order to evaluate the proposed method an experiment has been carried out. The method has been calculated for different images from the book of Brodatz 14, which are largely used as benchmark for texture analysis in computer vision and image processing literature. For the experiment, 40 classes of images with 10 samples each has been employed. Each image sample is a portion of $200 \times$ 200 pixels with 256 gray levels extracted from the original Brodatz image. One example of each of these 40 classes is showed in Figure 3.

The analysis step has been carried out applying over the texture signatures a Linear Discriminant Analysis (LDA), a well known method for estimating a linear subspace with good discriminative properties [11. The idea of the method is to find a feature space where the data projection presents variance between the classes larger than the variance within the classes. LDA is a supervised method, 
so it needs the class definitions for the estimation process [1112. A leave-one-out cross-validation scheme has been also employed.

An important issue in the method is the threshold set used to compute the texture signatures. The threshold set that better discriminate a texture set is that one which considers characteristics of the textures under analysis. So, a threshold selection approach has been used as follows:

1. For each texture in the database, compute its respective histogram;

2. Compute the mean histogram from the histograms of all textures. This mean histogram shows how the grays levels are distributed over the textures in the entire database;

3. Select $N$ different threshold levels by applying the Otsu's method of multilevel threshold [15]16] over the mean histogram;

This approach allows us to select the thresholds values where the betweenclass variance of the different gray levels in the database is maximum.

\section{Results}

To achieve a threshold set that better discriminate a texture is a difficult task. The choice of the thresholds values, as also the quantity of threshold values, depends on the characteristics presents in the textures under analysis. Multilevel Otsu's method is a reliable way to choose the optimal thresholds of an texture image by maximizing the between-class variance with an exhaustive search [1516]. When applied over the mean histogram of the texture database, multilevel Otsu's method furnishes a thresholds set related to the gray-levels distribution of the entire database, which allows to compose a more efficient texture signature $\psi(A)$.

Figure 4 shows the success rate of the proposed method as a function of the number of thresholds $N$ computed by multilevel Otsu's method. As expected, we note a increase in the success rate as we increase the number of thresholds levels used to composed the texture signature $\psi(A)$. This is due to the additional information about the texture complexity that is added to the signature vector. Each threshold value represents a gray-level where the homogeneity of the texture changes. Complexity analysis methods, such Fractal Dimension, measures the homogeneity of an image through its complexity. Multilevel Otsu's method combined with Fractal Dimension method allows to create a signature vector which describes the most relevant changes in texture complexity. In fact, using 70 threshold levels, the proposed method was able to classify correctly $97.00 \%$ of the texture samples.

Figure 5] shows the texture complexity as a function of the threshold for four given texture samples of two different texture classes. Although fractal dimension value decreases as we increase the applied threshold, it is important to note that it occurs at different rates for each texture class. It evidences the use of the proposed feature vector $\psi(A)$ as a feasible texture signature.

For comparison intentions, the proposed method have been compared with traditional texture analysis methods found in the literature. These descriptors are: Fourier descriptors [17], Co-ocorrence matrices [18] and Gabor filters 


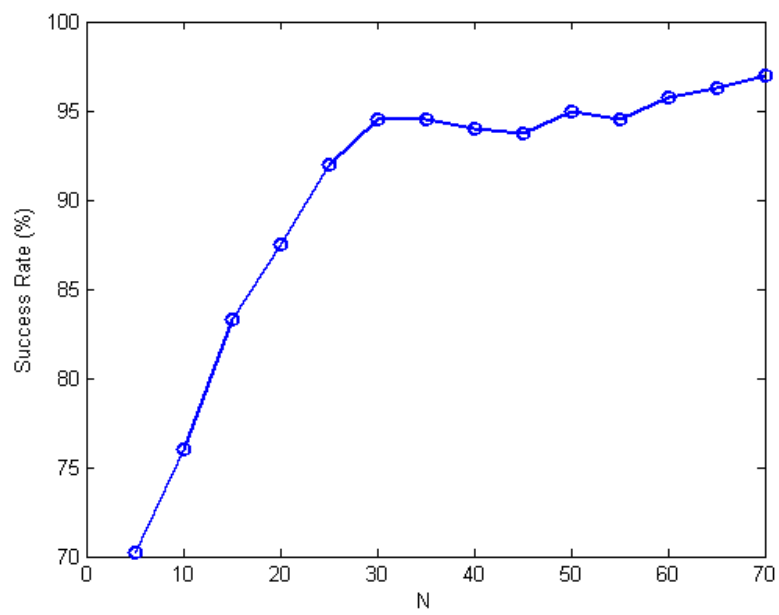

Fig. 4. Success rate as a function of the number of threshold levels $(N)$ considered. Best classification $(97.00 \%)$ is achieved when using $N=70$.

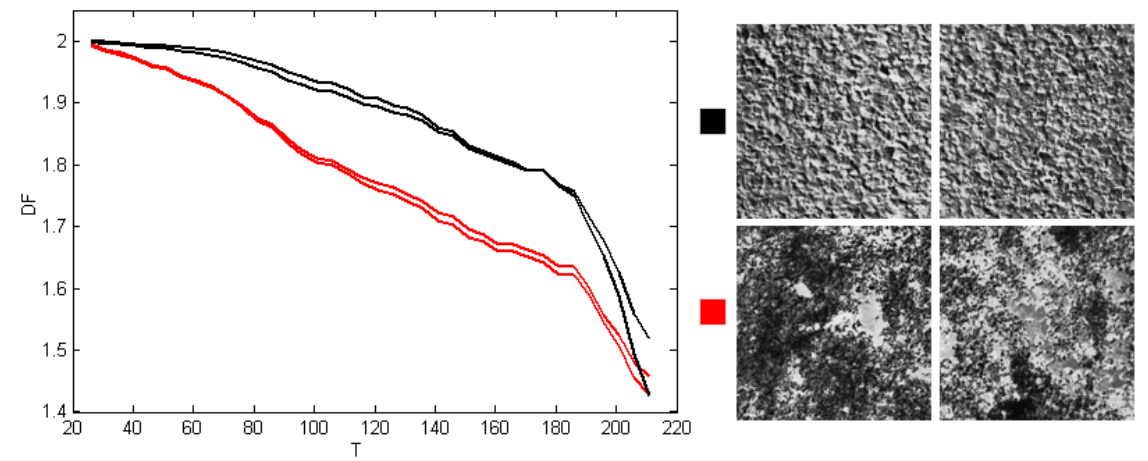

Fig. 5. Example of signatures computed for two different texture classes

\begin{tabular}{l|l|l|l|l|l}
19 & 20 & 21
\end{tabular} . Several different versions have been found on literature for these methods, however this paper considers just their conventional implementation.

Fourier descriptors: in the experiments, Fourier descriptors for texture analysis are composed of a feature vector sufficing the energy of the 99 most meaningful coefficients of the Fourier Transform. As low frequencies are placed at the center of the spectrum (after performing a shifting operation), each one of the coefficients corresponds to the sum of the spectrum absolute values placed to a radial distance from the center of the bi-dimensional transformation.

Co-ocorrence matrices: basically, they are joint probability distributions between pairs of pixels at a determined distance and direction. In this paper 
Table 1. Comparison results for different texture methods

\begin{tabular}{ccc}
\hline Method & Images correctly classified Success rate (\%) \\
\hline Co-ocorrence matrices & 330 & 82.50 \\
Fourier & 351 & 87.75 \\
Gabor Filters & 381 & 95.25 \\
Proposed Method & 388 & 97.00 \\
\hline
\end{tabular}

approach, distances of 1 and 2 pixels with angles of $-45^{\circ}, 0^{\circ}, 45^{\circ}, 90^{\circ}$ were used. Energy and entropy were computed from resulting matrices, totalizing a set of 16 descriptors. A non-symmetric version has been adopted in experiments.

Gabor filters: The 2-D Gabor filter is, basically, a bi-dimensional gaussian function moduled with an oriented sinusoid in a determined frequency and direction. This procedure consists of convolving an input image by a family of Gabor filters, which presents several scales and orientations of the same original configuration. Among numerous tests performed, the best results were achieved using a family of 16 filters ( 4 rotation filter and 4 scale filters), with frequency lower than 0.01 and superior than 0.3. Definition of the individual parameters of each filter follows mathematical model presented in 22 .

Table 1 shows the yielded results presented by each method. We realize that, though the proposed method uses a simple texture discrimination approach, the yielded results overcome traditional texture methods. Moreover, the proposed method also presents the lowest error rate for class, what indicates a great capacity of discriminating different texture classes.

\section{Conclusion}

We have presented a new approach of texture feature extraction based on the Box-Counting fractal dimension. The method uses several threshold values applied over a texture image to compute the changes in its complexity. Thresholds are computed using the Otsu's method for multilevel threshold, which allows to choose the optimal thresholds by maximizing the between-class variance. The proposed approach outputs a curve strictly related to the configuration of the texture and, consequently, to its complexity. An experiment using the proposed signature and linear discriminant analysis to classify a set of Brodatz texture have been performed. The results show the great potential of the method to be used as a texture analysis methodology.

\section{Acknowledgements}

A.R.B. acknowledges support from FAPESP (2006/54367-9). O.M.B. acknowledges support from CNPq (303746/2004-1). 


\section{References}

1. Ebert, D., Musgrave, K., Peachey, D., Perlin, K., Worley.: Texturing and Modeling: A Procedural Approach. Academic Press, London (1994)

2. Wang, L., Liu, J.: Texture classification using multiresolution markov random field models. Pattern Recognition Letters 20(2), 171-182 (1999)

3. Unser, M.: Texture classification and segmentation using wavelet frames. IEEE Trans. Image Processing 4(11), 1549-1560 (1995)

4. Emerson, C.W., Lam, N.N., Quattrochi, D.A.: Multi-scale fractal analysis of image texture and patterns. Photogrammetric Engineering and Remote Sensing 65(1), 51-62 (1999)

5. Chaudhuri, B.B., Sarkar, N.: Texture segmentation using fractal dimension. IEEE Trans. Pattern Anal. Mach. Intell 17(1), 72-77 (1995)

6. Schroeder, M.: Fractals, Chaos, Power Laws: Minutes From an Infinite Paradise. W.H. Freeman, New York (1996)

7. Tricot, C.: Curves and Fractal Dimension. Springer, Heidelberg (1995)

8. Lange, G.D., Marks, W.B.: Fractal methods and results in cellular morphology dimensions, lacunarity and multifractals. Journal of Neuroscience Methods 69(2), 123-136 (1996)

9. Coelho, R.C., Costa, L.F.: The box-counting fractal. dimension: Does it provide an accurate subsidy for experimental shape characterization? if so, how to use it? In: Anais do Sibgrapi, vol. 95, pp. 183-191 (1995)

10. Li, J., Sun, C., Du, Q.: A new box-counting method for estimation of image fractal dimension. In: International Conference on Image Processing, pp. 3029-3032 (2006)

11. Everitt, B.S., Dunn, G.: Applied Multivariate Analysis, 2nd edn. Arnold (2001)

12. Fukunaga, K.: Introduction to Statistical Pattern Recognition, 2nd edn. Academic Press, London (1990)

13. Falconer, K.J.: Fractal geometry: mathematical foundations and applications, 288 p. Wiley, Chichester (1990); CALL NUM: QA614.86 .F35 1990

14. Brodatz, P.: A Photographic Album for Arts and Design, vol. 1. Dover Publishing Co., Toronto (1966)

15. Liao, P.S., Chen, T.S., Chung, P.C.: A fast algorithm for multilevel thresholding. J. Inf. Sci. Eng 17(5), 713-727 (2001)

16. Otsu, N.: A threshold selection method from gray level histograms. IEEE Trans. Systems, Man and Cybernetics 9, 62-66 (1979); minimize intra and inter class variance

17. Azencott, R., Wang, J.P., Younes, L.: Texture classification using windowed fourier filters. IEEE Trans. Pattern Anal. Mach. Intell. 19(2), 148-153 (1997)

18. Haralick, R.M.: Statistical and structural approaches to texture. Proc. IEEE 67(5), 786-804 (1979)

19. Jain, A.K., Farrokhnia, F.: Unsupervised texture segmentation using Gabor filters. Pattern Recognition 24(12), 1167-1186 (1991)

20. Daugman, J., Downing, C.: Gabor wavelets for statistical pattern recognition. In: Arbib, M.A. (ed.) The Handbook of Brain Theory and Neural Networks, pp. 414419. MIT Press, Cambridge (1995)

21. Idrissa, M., Acheroy, M.: Texture classification using gabor filters. Pattern Recognition Letters 23(9), 1095-1102 (2002)

22. Manjunath, B.S., Ma, W.Y.: Texture features for browsing and retrieval of image data. IEEE Trans. Pattern Anal. Mach. Intell 18(8), 837-842 (1996) 